\title{
The Modern Landscape of Endocrine Therapy for Premenopausal Women with Breast Cancer
}

\author{
Lorenzo Rossi Olivia Pagani \\ Institute of Oncology of Southern Switzerland (IOSI) and Breast Unit of Southern Switzerland (CSSI), Bellinzona, Switzerland
}

\section{Keywords}

Premenopausal - Breast cancer - Endocrine therapy . Ovarian suppression - Aromatase inhibitors - Tamoxifen

\section{Summary}

The optimal endocrine therapy for premenopausal women with early and advanced breast cancer still remains an important and controversial issue. For over 30 years, tamoxifen has been the gold standard in the adjuvant setting. New therapeutic options, such as the addition of ovarian function suppression to oral endocrine therapy (either tamoxifen or aromatase inhibitors), can improve outcomes over tamoxifen alone in well-selected patients. Treatment duration has also been revisited, and extended therapy is becoming a new standard of care, especially in high-risk patients. Endocrine therapy for advanced disease still represents a challenge and a research priority. New drugs and combinations able to overcome endocrine resistance are at the horizon, and their role in premenopausal women should be better elucidated. Side effects and quality of life (including family planning considerations) play an important role in treatment selection and in the patients' treatment adherence and should always be discussed before start of treatment. The paper will specifically focus on how to integrate all new treatment options in the current armamentarium of endocrine therapy of premenopausal women, with the aim of best fine-tuning treatment selections according to the individual risk/benefit evaluation.

(C) 2015 S. Karger GmbH, Freiburg

\section{Setting the Scene}

Hormone receptor-positive $(\mathrm{HR}+)$ breast cancer $(\mathrm{BC})$ is the most common histological subtype across all age groups [1], even if the proportion of $\mathrm{HR}+\mathrm{BC}$ is known to be correlated with age. A large Danish study (26,944 patients) [2] reported a direct association between $\mathrm{HR}+\mathrm{BC}$ and age (from $51.4 \%$ in patients $<35$ years to $67.6 \%$ in women $40-44$ years), and other studies show similar figures $[3,4]$. In developed countries, the majority of young women (i.e. < 40 years) with $\mathrm{HR}+\mathrm{BC}$ have early disease at diagnosis: In the last decades, long-term survival with modern therapies has dramatically improved, also for patients with advanced disease $[5,6]$.

\section{Is There a Standard Adjuvant Endocrine Treatment?}

Several therapeutic options are currently available and the individual choice should consider the risk of recurrence, the latest scientific evidence as well as the toxicity profile, the patient's comorbidities, and her personal preference.

The first-ever endocrine therapy (ET) in premenopausal patients was ovarian suppression (OFS) by surgical castration or ovarian irradiation [7]. In developed countries, this approach has been progressively replaced by gonadotropin-releasing hormone analogues (GnRHas), with comparable results [8]. Surgical castration remains a low-cost choice in developing countries. Bilateral salpingo-oophorectomy is also a valid alternative in BRCA1/2 mutation carriers with completed family planning.

For over 30 years, tamoxifen for 5 years has been the gold standard [9-11]. The benefit of adjuvant tamoxifen is independent of age [12], with a $30 \% \mathrm{BC}$ mortality reduction at 15 years. In women $<50$ years, the greatest benefit is achieved in patients with axillary involvement [13].

Aromatase inhibitors (AIs) block the conversion of androgens to estrogens, thereby reducing tissue and plasmatic estrogen levels [14]. Third-generation AIs such as letrozole and anastrozole (nonsteroidal) and exemestane (steroidal) are associated with a modest but significant improvement in disease outcomes over tamoxifen in menopausal women [15]. AIs cannot be used alone in premenopausal patients due to the risk of ovarian stimulation resulting in a paradoxical increase in circulating estrogens.

\section{KARGER \\ Fax +497614520714

\section{() 2015 S. Karger GmbH, Freiburg}

$1661-3791 / 15 / 0105-0312 \$ 39.50 / 0$
Lorenzo Rossi, MD

Oncological Institute of Southern Switzerland

Via Ospedale

6500 Bellinzona, Switzerland

Lorenzo.Rossi@eoc.ch 
In the International Breast Cancer Study Group (IBCSG)-led Suppression of Ovarian Function Trial (SOFT), 3,066 premenopausal women, stratified according to prior chemotherapy, were randomized to 5 years of tamoxifen, tamoxifen plus OFS, or exemestane plus OFS. Overall, after a median follow-up of 5.6 years, adding OFS to tamoxifen did not provide a significant benefit in terms of disease-free survival (DFS) $(84.7 \%$ in the tamoxifen group, $86.6 \%$ in the tamoxifen/OFS group; hazard ratio (HR) 0.83 , 95\% confidence interval (CI) 0.66-1.04; P = 0.10) [16]. However, after in-depth data analysis, different scenarios emerge according to the administration of chemotherapy. Among low-risk patients who did not receive chemotherapy (mostly $>40$ years, with small, node-negative tumors of low-intermediate grade), > 95\% remained free from $\mathrm{BC}$ at 5 years irrespective of treatment assignation. In contrast, in the cohort of patients at higher risk of relapse who needed chemotherapy according to the treating physician and remained premenopausal afterwards, the rate of freedom from BC at 5 years was significantly higher among patients receiving tamoxifen plus OFS than in those receiving tamoxifen alone $(82.5 \%$ and $78.0 \%$, respectively; HR $0.78,95 \%$ CI $0.60-1.02)$. Of note, in the subset of very young patients ( $<35$ years), BC recurred in approximately one-third of the patients receiving tamoxifen alone and in one-sixth of those treated with exemestane plus OFS $(67.7 \%$ and $83.4 \%$, respectively), suggesting that OFS plays a major role in younger premenopausal patients.

The recent combined analysis of the TEXT and SOFT trials also showed that adjuvant exemestane plus OFS, compared to tamoxifen plus OFS, significantly reduced the relative risk of invasive BC recurrence by $34 \%$ [17]. The absolute $3.8 \%$ DFS benefit is comparable with the results from randomized trials of adjuvant AIs versus tamoxifen in postmenopausal women. Of note, chemotherapy was not administered to 1,996 women (43\%), and $21 \%$ of the TEXT patients who did not receive chemotherapy had node-positive disease. In this subgroup, the rate of freedom from BC at 5 years was $>$ $97 \%$ among those women who received combined exemestane plus OFS, suggesting an excellent prognosis with highly effective adjuvant $\mathrm{ET}$ alone.

Both trials, SOFT and TEXT, showed no significant differences in overall survival (OS): Conclusions are premature at this time point in this patient population, after $4.1 \%$ of the patients have died.

Overall, the results of the SOFT and TEXT trials indicate that the outcome of premenopausal women with $\mathrm{HR}+$ early $\mathrm{BC}$ receiving modern adjuvant therapy is very good and provide a practicechanging, better tailored treatment algorithm (fig. 1).

The Austrian Breast and Colorectal Cancer Study Group-12 (ABCSG-12) trial also investigated OFS plus either tamoxifen or the AI anastrozole. After a median follow-up of 94.4 months there is no DFS benefit and a non-significantly higher risk of death (HR $=1.63,95 \% \mathrm{CI} 1.05-1.45 ; \mathrm{P}=0.030)$ in the anastrozole-treated group [18]. These apparently divergent results can be explained by several differences in the ABCSG-12 trial as compared to SOFT and TEXT: Only $5 \%$ of the patients received chemotherapy; the trial had a smaller statistical power (three times lower number of
Endocrine Therapy on Premenopausal HR+ early BC: a proposal

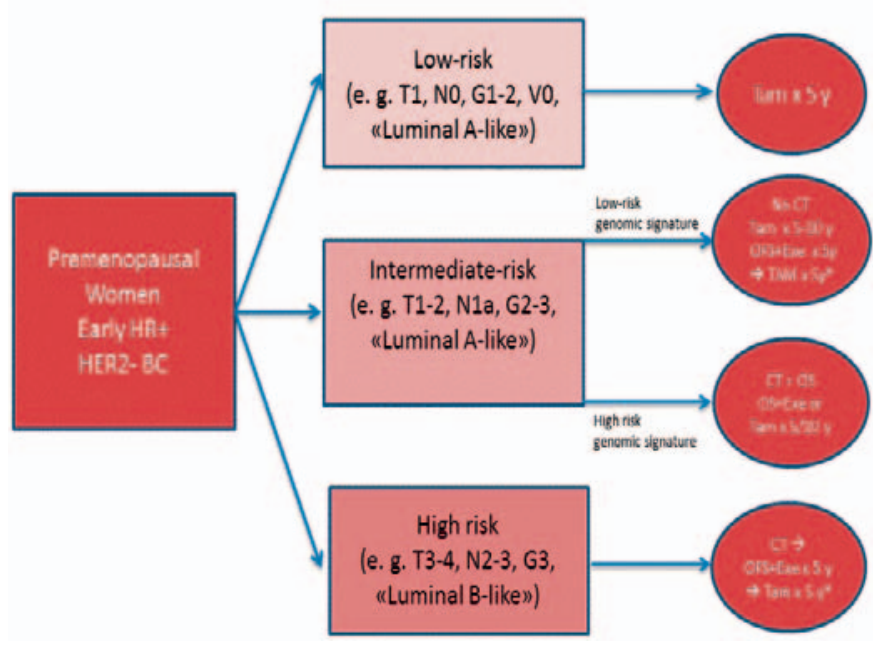

Fig. 1. Suggested flow chart for ET of premenopausal patients with HR+ early BC. $\mathrm{HR}+=$ Hormone-positive tumor, $\mathrm{BC}=$ breast cancer, TAM $=$ tamoxifen, $\mathrm{CT}=$ chemotherapy, $\mathrm{OFS}=$ ovarian function suppression, $\mathrm{EXE}=$ exemestane. ${ }^{*}$ Note: Some of these forms of treatment are suggestions and do not represent the standard of care.

events); the treatment duration was only 3 years; the use of zoledronic acid and the influence of overweight might have abrogated the effect of the AI.

The last St Gallen consensus also tried to best fine-tune ET in different clinical scenarios [19]. The panel considered several factors in favor of adding OFS to oral ET: age $\leq 35$ years, premenopausal estrogen levels after adjuvant chemotherapy, and $\geq 4$ positive axillary nodes. The use of an AI, rather than tamoxifen, plus OFS was greatly preferred in case of $\geq 4$ positive nodes, with fewer panelists valuing age $\leq 35$ years, grade 3 , and premenopausal estrogen levels after adjuvant chemotherapy.

\section{Which Is the Optimal Duration of Adjuvant ET?}

The standard duration of tamoxifen is at least 5 years [9]. Despite small old trials $[20,21]$ concluded, there is no added benefit to prolong ET beyond 5 years; the most recent and sound data from the ATLAS (Adjuvant Tamoxifen: Longer Against Shorter) and aTTom (adjuvant Tamoxifen - To offer more?) trials in almost 20,000 women showed that continuing tamoxifen to 10 years provides a further reduction in both disease recurrence and mortality [22, 23].

The ATLAS trial randomized 15,244 pre- and postmenopausal women to 10 versus 5 years of adjuvant tamoxifen. After a median follow-up of 7.6 years, 10 years of tamoxifen reduced the $\mathrm{BC}$ recurrence by $3 \%$ ( $18 \%$ vs. $21 \%$; relative risk (RR) 0.84 , CI $95 \% 0.76-$ 0.94). The protective effect extends well over the 10 -year treatment period (RR 0.90, 95\% CI 0.79-1.02 during years 5-9; RR 0.70, 95\% CI 0.62-0.90 during subsequent years), regardless of age and nodal status. Overall, patients on extended therapy had an increased risk of endometrial cancer (RR 1.74, 95\% CI 1.30-2.34; P = 0.0002) and 
pulmonary embolism (RR 1.87, 95\% CI 1.13-3.07; P = 0.01). In premenopausal women, the risk of tamoxifen-related uterine cancer or vascular side effects is very low and largely counterbalanced by the great absolute reduction in BC mortality. In the aTTom trial, 7,000 women were included and randomized to 10 versus 5 years of adjuvant tamoxifen. Despite the fact that the HR status was not available in a consistent proportion of patients, the longertreatment group had fewer BC recurrences compared with the 5 -year treatment group (28\% versus $32 \% ; \mathrm{P}=0.003$ ). Tamoxifen for 10 years also reduced the BC mortality compared with 5 years of treatment (392 versus 443 deaths; $21 \%$ vs. $24 \%$; $\mathrm{P}=0.06$ ). Overall, these results can be considered as practice changing, especially for patients at high risk of recurrence.

In the TEXT and SOFT trials, the duration of both oral ET and OFS was 5 years. To date, there are no data on the extension of adjuvant oral ET (either AIs or tamoxifen) plus OFS beyond 5 years in premenopausal patients.

The National Cancer Institute of Canada Clinical Trials Group (NCIC-CTG) MA.17/Breast International Group (BIG) 1-97 trial reported the best DFS benefit of 5 years of letrozole after 5 years of tamoxifen (HR 0.25, 95\% CI 0.12-0.51) in premenopausal women at diagnosis who became definitively postmenopausal at the time of randomization [24].

\section{Metastatic Disease}

In case of indolent $\mathrm{HR}+$ metastatic disease, ET represents the upfront therapeutic option [25], with an expected disease response of $30-40 \%$ after $2-3$ months of treatment and a median response duration of 12-18 months [26]. Acceptable first-line options include tamoxifen or AIs plus OFS [25]. In the PALOMA-3 trial [27], the combination of the cyclin-dependent kinase (CDK)4-CDK6 inhibitor palbociclib plus fulvestrant, in patients relapsed or progressed during prior ET, resulted in longer progression-free survival (PFS) than fulvestrant alone, with a similar relative difference in pre- and/or perimenopausal and postmenopausal patients (HR for disease progression or death, 0.44 and 0.41 , respectively). Premenopausal or perimenopausal women also received goserelin. Exemestane plus everolimus has not yet been studied in young women.

\section{Side Effects and Survivorship Issues}

ET in premenopausal patients is generally associated with menopausal side effects, e.g. hot flushes, vaginal dryness, and mood swings. Iatrogenic menopausal symptoms are generally stronger and faster than symptoms after natural menopause, but treatment tolerance is very subjective. Side effects are also greatly dependent on the class of drugs administered. GnRHas can cause more severe bone loss, hot flushes, dyslipidemia, and sexual dysfunction than tamoxifen [28]. On the other hand, tamoxifen is characterized by vaginal discharge, thromboembolic events, phlebitis, irregular menses, vaginal bleeding, endometrial polyps, and ovarian cysts $[29,30]$. A meta-analysis of 23 trials (45,936 patients) showed that tamoxifen was associated with a statistically significant increase in endometrial cancer risk (RR 2.70, CI 95\% 1.94-3.75) [31]. The risk is higher in postmenopausal patients. In menopausal women, tamoxifen is associated with a decrease of low-density lipoproteins and total cholesterol and an increase in bone mineral density. These effects are not evident in premenopausal patients [32-34]: As a consequence, bone health should be promoted and checked in young women taking the drug. Estrogen deprivation is also associated with cognitive impairment, in particular in verbal memory and executive functioning [35].

In addition, the increasing indication for extended adjuvant ET does not help the former patient to feel healthy and cured and can compromise family relationships and work commitments.

\section{Pregnancy Desire}

BC in young women often occurs before completion of any reproductive plans; all the retrospective evidence suggests that pregnancy after BC is safe for the mother and the offspring [36, 37]. In a multicenter retrospective cohort study, patients who became pregnant any time after $\mathrm{BC}$ were matched to non-pregnant $\mathrm{BC}$ patients with similar HR and nodal status, adjuvant therapy, and age at diagnosis. The study showed no difference in DFS between pregnant and non-pregnant patients in the $\mathrm{HR}+$ population [38]. In the same analysis, also no difference in DFS was observed between patients who became pregnant $<2$ years following $\mathrm{BC}$ diagnosis and those who became pregnant afterwards. On the other hand, ET postpones family planning and can therefore compromise the chance to conceive. A prospective observational study of women with $\mathrm{BC}$ diagnosed $<40$ years ( $\mathrm{HOHO}$ study, 'Helping Ourselves Helping Others') showed that $20 \%$ of patients desire children after $\mathrm{BC}$ and are willing to take $<5$ years of tamoxifen [39]. An IBCSGcoordinated prospective trial (POSITIVE for Pregnancy Outcome and Safety of Interrupting Therapy for women with endocrine responsIVE breast cancer) is evaluating the risk of $\mathrm{BC}$ relapse associated with an interruption of ET to allow pregnancy and the factors associated with pregnancy success.

Patients should be sensitized to use non-hormonal contraception during ET: Tamoxifen, despite amenorrhea, is associated with a high frequency of severe congenital abnormalities in the offspring [40], and sustained ovarian suppression is not achieved in up to $20 \%$ of patients under GnRHas [41].

\section{Future Perspectives and Challenges}

Despite significant recent improvements, both healthcare professionals and patients still face several challenges in the future. One of the most striking and urgent needs is to learn how to incorporate the information achieved during modern randomized clinical trials (in early and advanced disease settings) into the individ- 
ual routine patient care. Improving quality of life and reducing side effects is also pivotal in order to best exploit, e.g., the use of AIs and extended therapy in patients with early disease. Furthermore, considering the high number of young women living with advanced $\mathrm{BC}$ worldwide, we must develop new strategies and drugs as firstline treatment and to overcome resistance in pretreated patients.

\section{Disclosure Statement}

The authors declare that there are no conflicts of interest.

\section{References}

1 Keegan TH, DeRouen MC, Press DJ, et al.: Occurrence of breast cancer subtypes in adolescent and young adult women. Breast Cancer Res 2012;14:R55.

2 Bentzon N, Düring M, Rasmussen BB, et al.: Prognostic effect of estrogen receptor status across age in primary breast cancer. Int J Cancer 2008;122:1089-1094.

3 Collins LC, Marotti JD, Gelber S, et al.: Pathologic features and molecular phenotype by patient age in a large cohort of young women with breast cancer. Breast Cancer Res Treat 2012;131:1061-1066.

4 Anders CK, Hsu DS, Broadwater G, et al.: Young age at diagnosis correlates with worse prognosis and defines a subset of breast cancers with shared patterns of gene expression. J Clin Oncol 2008;26:3324-3330.

5 Foukakis T, Fornander T, Lekberg T, et al.: Age-specific trends of survival in metastatic breast cancer: 26 years longitudinal data from a population-based cancer registry in Stockholm, Sweden. Breast Cancer Res Treat 2011;130:553-560.

6 Tjokrowidjaja A, Lee CK, Houssami N, Lord S: Metastatic breast cancer in young women: a populationbased cohort study to describe risk and prognosis. Intern Med J 2014;44:764-770.

7 Ravdin RG, Lewison EF, Slack NH, et al.: Results of a clinical trial concerning the worth of prophylactic oophorectomy for breast carcinoma. Surg Gynecol Obstet 1970;131:1055-1064.

8 Early Breast Cancer Trialists' Collaborative Group: Ovarian ablation in early breast cancer: overview of the randomised trials. Lancet 1996;348:1189-1196.

9 Partridge AH, Pagani O, Abulkhair O, Aebi S, Amant F, Azim HA Jr, Costa A, Delaloge S, Freilich G, Gentilini OD, Harbeck N, Kelly CM, Loibl S, Meirow D, Peccatori F, Kaufmann B, Cardoso F: First international consensus guidelines for breast cancer in young women (BCY1). Breast 2014;23:209-220.

10 Goldhirsch A, Winer EP, Coates AS, Gelber RD, Pic cart-Gebhart M, Thurlimann B, et al.: Personalizing the treatment of women with early breast cancer: highlights of the St Gallen International Expert Consensus on the Primary Therapy of Early Breast Cancer 2013. Ann Oncol 2013;24:2206-2223.

11 Senkus E, Kyriakides S, Penault-Llorca F, Poortmans P, Thompson A, Zackrisson S, Cardoso F: Primary breast cancer: ESMO clinical practice guidelines for diagnosis, treatment and follow-up. Ann Oncol 2013; 24(suppl 6):vi7-vi23.

12 Early Breast Cancer Trialists' Collaborative Group (EBCTCG), Davies C, Godwin J, Gray R, et al.: Relevance of breast cancer hormone receptors and other factors to the efficacy of adjuvant tamoxifen: patientlevel meta-analysis of randomised trials. Lancet 2011 378:771-784.
13 Early Breast Cancer Trialists' Collaborative Group (EBCTCG): Effects of chemotherapy and hormonal therapy for early breast cancer on recurrence and 15year survival: an overview of the randomised trials. Lancet 2005;365:1687-1717.

14 Smith IE, Dowsett M: Aromatase inhibitors in breast cancer. N Engl J Med 2003;348:2431-2442.

15 Dowsett M, Cuzick J, Ingle J, et al.: Meta-analysis of breast cancer outcomes in adjuvant trials of aromatase inhibitors versus tamoxifen. J Clin Oncol 2010;28: 509-518.

16 Francis PA, Regan MM, Fleming GF, et al.: Adjuvant ovarian suppression in premenopausal breast cancer. N Engl J Med 2015;372:436-446.

17 Pagani O, Regan MM, Walley BA, et al.: Adjuvant exemestane with ovarian suppression in premenopausal breast cancer. N Engl J Med 2014;371:107-118.

18 Gnant M, Mlineritsch B, Stoeger H, et al.: Zoledronic acid combined with adjuvant endocrine therapy of tamoxifen versus anastrozol plus ovarian function suppression in premenopausal early breast cancer: final analysis of the Austrian Breast and Colorectal Cancer Study Group Trial 12. Ann Oncol 2015;26:313-320.

19 Coates AS, Winer EP, Goldhirsch A, et al.: Tailoring therapies - improving the management of early breast cancer: St Gallen International Expert Consensus on the Primary Therapy of Early Breast Cancer 2015. Ann Oncol 2015;26:1533-1546.

20 Fisher B, Dignam J, Bryant J, et al.: Five versus more than five years of tamoxifen for lymph node-negative breast cancer: updated findings from the National Surgical Adjuvant Breast and Bowel Project B-14 randomized trial. J Natl Cancer Inst 2001;93:684-690.

21 Stewart HJ, Forrest AP, Everington D, et al.: Randomised comparison of 5 years of adjuvant tamoxifen with continuous therapy for operable breast cancer. The Scottish Cancer Trials Breast Group. Br J Cancer 1996;74:297-299.

22 Davies C, Pan H, Godwin J, et al.: Long-term effects of continuing adjuvant tamoxifen to 10 years versus stopping at 5 years after diagnosis of oestrogen receptorpositive breast cancer: ATLAS, a randomised trial. Lancet 2013;381:805-816.

23 Gray RG, Rea D, Handley K, et al.: aTTom: Long-term effects of continuing adjuvant tamoxifen to 10 years versus stopping at 5 years in 6,953 women with early breast cancer. J Clin Oncol 2013;31(suppl):abstr 5.

24 Higgins MJ, Liedke PE, Goss PE: Extended adjuvant endocrine therapy in hormone dependent breast cancer: the paradigm of the NCIC-CTG MA.17/BIG 1-97 trial. Crit Rev Oncol Hematol 2013;86:23-32.

25 Cardoso F, Costa A, Norton L, et al.: ESO-ESMO 2nd international consensus guidelines for advanced breast cancer (ABC2). Breast 2014;23:489-502.

26 Bernanrd-Marty C, Cardoso F, Piccart MJ: Facts and controversies in systemic treatment of metastatic breast cancer. Oncologist 2004;9:617-632.
27 Turner NC, Ro J, André F, et al.: Palbociclib in hormone-receptor-positive advanced breast cancer. N Engl J Med 2015;373:209-219.

28 Kafmann M, Jonat W, Kleeberg U, et al.: Goserelin, a depot gonadotrophin-releasing hormone agonist in the treatment of premenopausal patients with metastatic breast cancer. J Clin Oncol 1989;7:1113-1119.

29 Baum M: Has tamoxifen had its day? Breast Cancer Res 2002;4:213-217.

30 Colleoni M, Giobbie-Hurder A: Benefits and adverse effects of endocrine therapy. Ann Oncol 2010;21 (suppl 7):vii107-viil11.

31 Braithwaite RS, Chlebowski RT, Lau J, et al: Metaanalysis of vascular and neoplastic events associated with tamoxifen. J Gen Intern Med 2003;18:937-947.

32 Ramaswamy B, Shapiro CL: Osteopenia and osteoporosis in women with breast cancer. Semin Oncol 2003; 30:763-775.

33 Love RR, Wiebe DA, Feyzi JM, et al.: Effects of tamoxifen on cardiovascular risk factors in postmenopausal women after 5 years of treatment. J Natl Cancer Inst 1994;86:1534-1539.

34 Guetta V, Lush RM, Figg WD, et al.: Effects of the antiestrogen tamoxifen on low density lipoprotein concentrations and oxidation in post-menopausal women. Am J Cardiol 1995;76:1072-1073.

35 Paganini-Hill A, Clark JL: Preliminary assessment of cognitive function in breast cancer patients treated with tamoxifen. Breast Cancer Res Treat 2000;64:175176.

36 De Bree E, Makrigiannakis A, Askoxylakis J: Pregnancy after breast cancer. A comprehensive review. J Surg Oncol 2010;101:534-542.

37 Pagani O, Partridge A, Korde L, et al.: Pregnancy after breast cancer: if you wish, ma'am. Breast Cancer Res Treat 2011;129:309-317.

38 Azim HA Jr, Kroman N, Paesmans N, et al.: Prognostic impact of pregnancy after breast cancer according to estrogen receptor status: a multicenter retrospective study. J Clin Oncol 2013;31:73-79.

39 Ruddy KJ, Gelber SI, Tamimi RM, et al.: Prospective study of fertility concerns and preservation strategies in young women with breast cancer. J Clin Oncol 2014; 32:1151-1156.

40 Braems G, Denys H, De Wever O, et al.: Use of tamoxifen before and during pregnancy. Oncologist 2011;16: 1547-1551.

41 Bellet M, Gray KP, Francis PA, et al.: Estrogen levels in premenopausal (prem) patients (pts) with hormonereceptor positive $(\mathrm{HR}+)$ early breast cancer $(\mathrm{BC})$ receiving adjuvant triptorelin (Trip) plus exemestane (E) or tamoxifen (T) in the SOFT trial: SOFT-EST substudy. J Clin Oncol 2014;32(suppl 5s):abstr 585. 